\section{The Cavoatrial Anastomosis Procedure in Anomalous Connection of Superior Vena Cava to Left Atrium}

\author{
Rabin Gerrah, MD', Elizabeth Fonseca Escalante, MD', \\ Matthias Gorenflo, MD', and Tsvetomir Loukanov, MD' \\ ' Division of Pediatric Cardiac Surgery, Department of Cardiac \\ Surgery, Heidelberg University, Heidelberg, Germany
}

DOI: $10.1177 / 2150135119878102$

\begin{abstract}
Anomalous connection of the superior vena cava to the morphologic left atrium is a rare congenital systemic venous abnormality. As opposed to pulmonary venous anomalies, the significant right-to-left shunt in these patients warrants a correction early in life. Optimal repair technique for combined pulmonary and systemic venous anomalies is not defined yet. Herein, we describe a neonate with such a diagnosis treated with cavoatrial anastomosis, known as Warden procedure with excellent results.
\end{abstract}

\section{Introduction}

The cavoatrial anastomosis, known as Warden procedure, was originally designed to repair the anomalous connection of the pulmonary vein (PV) to the superior vena cava (SVC) when baffling of the PV to the left atrium (LA) is a challenge. Herein, we present a case of a neonate in whom the cavoatrial anastomosis technique was used to repair a rare systemic venous drainage along with pulmonary venous anomaly.

\section{Case Report}

A one-week-old, 3-kg full-term newborn was admitted with cyanosis and hypoxemia (oxygen saturation $69 \%$ ). Further workup revealed anomalous connection of the right-sided SVC to the LA, presence of a superior sinus venosus defect with left-to-right shunt, patent foramen ovale, and patent ductus arteriosus in a situs solitus heart with no other malformations.

On day of life 25 , the patient was taken to operating room for correction of his combined anomalous systemic and pulmonary venous drainage. The anatomy was remarkable for anomalous connection of the SVC to the LA, anomalous connection of three minuscule PVs to anterolateral aspect of the SVC, and septal defects as described by echocardiogram (Figure 1; supplemental video). In this setting, the decision to perform a Warden procedure seemed the most appropriate. Initially, the ductus arteriosus was ligated. The SVC was mobilized and transected above the junction of the highest connecting PV and distal stump was over sewn. The right atrial (RA) appendage was explored with a horizontal incision and a direct anastomosis was constructed between the proximal end of the SVC and the RA appendage. This anastomosis could accommodate a $10-\mathrm{mm}$ Hegar dilator easily.

Trabeculations were resected to ensure unobstructed flow to the RA and to avoid late stenosis. This anastomosis diverted the SVC flow to the RA in a normal fashion. The sinus venosus septal defect was closed using an autologous pericardial patch, which baffled the flow from the anomalous PVs to the LA. The patent foramen ovale was closed primarily. This combined strategy of both Warden and patch repair achieved a normal anatomy of this complex dual venous drainage anomaly.

Upon completion of this repair, normal sinus rhythm, full oxygenation with saturation of $100 \%$, and normal RA pressures were observed. The postoperative course was remarkable for right diaphragmatic paresis with mild respiratory effect with no need for further treatment. The patient was discharged home on postoperative day 13 in good condition. In a six-month follow-up, the patient was in good condition; on echo, the SVC diameter was measured as $4 \mathrm{~mm}$ and the anastomosis was open with a diameter of $7 \mathrm{~mm}$.

\section{Comments}

Anomalous connection of the SVC to the LA is extremely rare, first reported in $1956^{1}$ and then in other reports. ${ }^{2-4}$ Unlike pulmonary venous anomalies that create a left-to-right shunt and often are diagnosed later in life, the anomalous SVC to LA creates a large right-to-left shunt by diverting the majority of upper body systemic venous drainage to the LA, and potentially cyanosis. The exact etiology of this lesion is not known; however, it is believed to be related to abnormal development of sinus venosus. During the embryogenesis, the abnormal resorption of the cephalic portion of the right sinus venosus valve possibly creates a seal that prevents the SVC connection to the RA, resulting in this anomaly. ${ }^{4}$ Due to physiologic significance of this anomaly, an early repair is warranted. Furthermore, beyond the problem of shunting, this lesion entails a potential of paradoxical embolism. For this reason, prior to correction of this anomaly, use of intravenous line in the upper extremity should be avoided to minimize the risk of systemic, especially cerebral emboli.

The Warden procedure is an adjunct in repair of sinus venosus defect when the high drainage of PV to SVC excludes the option of an easy repair with baffling the pulmonary venous flow to the LA by means of a patch that closes the defect. ${ }^{5}$ Performing a Warden procedure has generally been reserved for limited indications due to its potential for complications such as late stenosis (usually a result of insufficient resection and clearance of the trabeculations in the RA appendage) and sinoatrial node dysfunction with consequent dysrhythmia.

For this anomaly, the Warden procedure seems to be the most intuitive and reasonable repair, achieving nearly normal anatomy. The case of a four-year-old with some similarities to ours was reported by Recto et al; however, in that case, the SVC did not drain exclusively to the LA. ${ }^{6}$ Yerebakan described an unusual case in which the right SVC had connections to both atria; some elements of a Warden procedure were used to repair that anomaly. ${ }^{7}$ Braudo et al presented a case in which the right SVC pursued a tortuous course to connect to the LA,

Submitted April II, 2019; Accepted August 16, 2019.

\section{Corresponding Author:}

Rabin Gerrah, Division of Pediatric Cardiac Surgery, Department of Pediatric and Congenital Cardiac Surgery, Heidelberg University, INF II0, Heidelberg 69120, Germany.

Email: rgerrah@gmail.com 


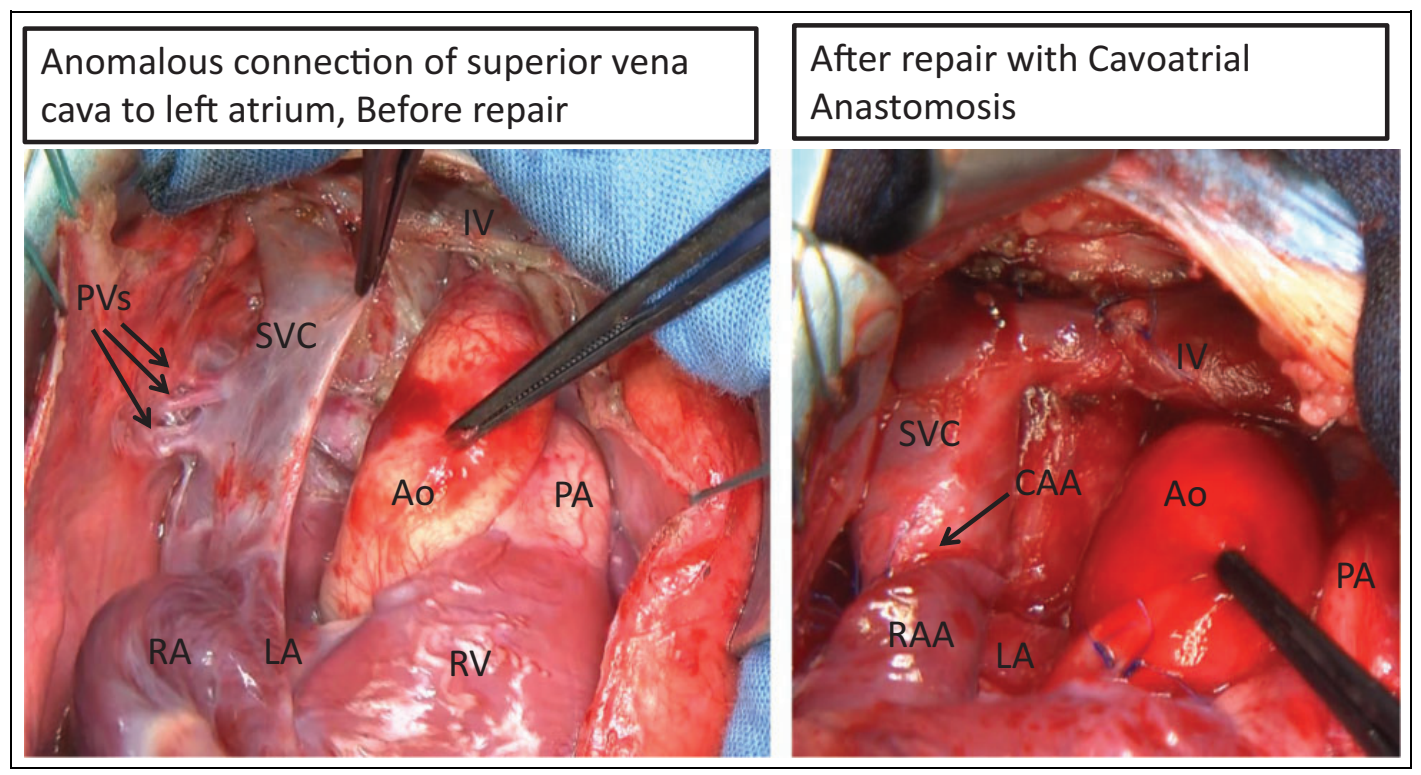

Figure I. Surgical view of the anomaly before and after repair with cavoatrial anastomosis. Note the connection of the superior vena cava to the superoposterior aspect of the left atrium. Ao indicates aorta; CAA, cavoatrial anastomosis; IV, innominate vein; $L A$, left atrium; PA, pulmonary artery; PVs, pulmonary veins; RA, right atrium; RV, right ventricle; SVC, superior vena cava.

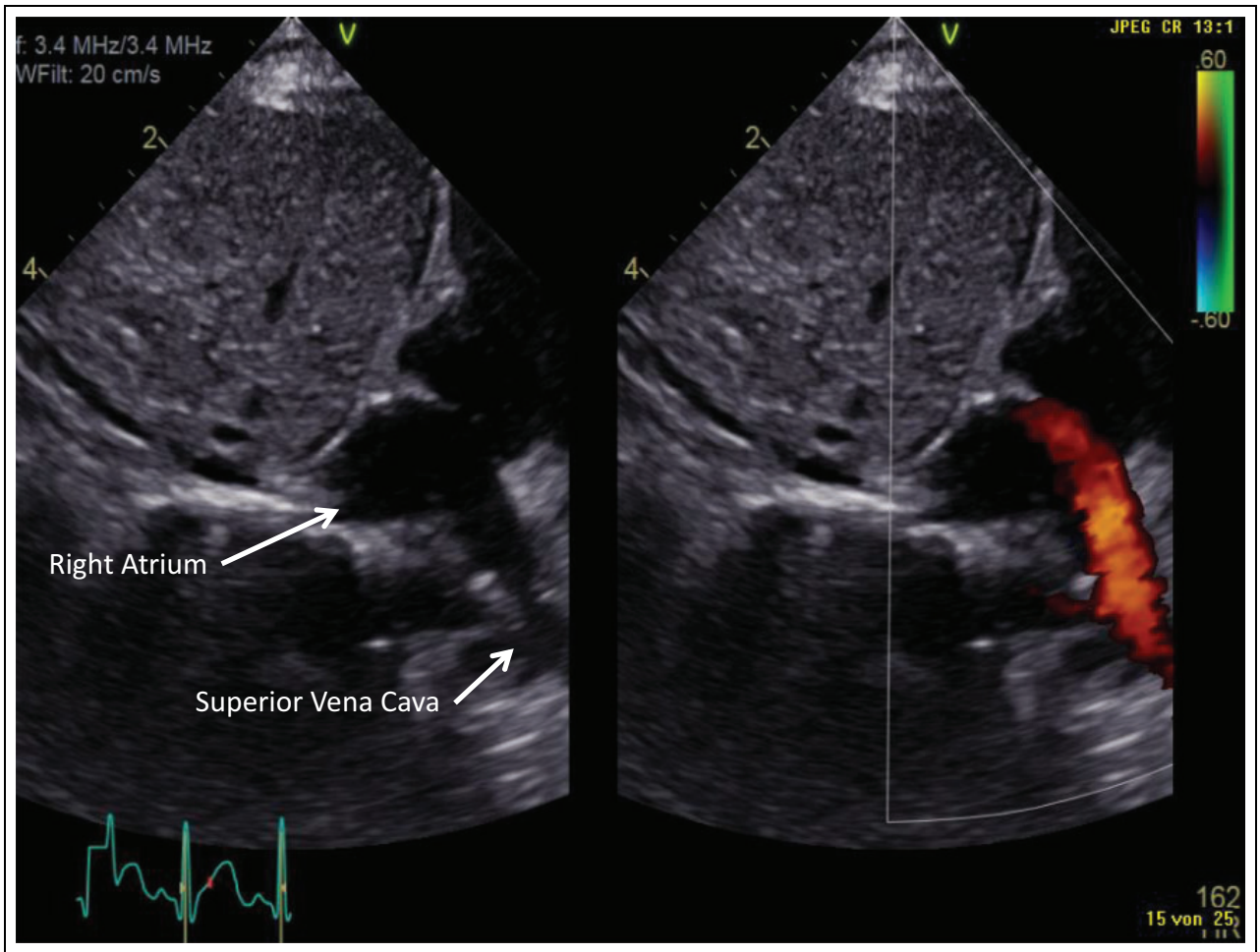

Figure 2. Postrepair

echocardiographic view of the repair at follow-up showing a wide open connection between the superior vena cava and right atrium.

and it was repaired by direct anastomosis of SVC to an L-shaped incision in the RA. ${ }^{4}$ Our case presents an ideal indication for the Warden procedure, and therefor expands its usage beyond the usual setting of repair of sinus venosus defects. On the latest postoperative follow-up, six months after the surgery, patient is doing well and echocardiography shows wide open anastomosis between the SVC and RA (Figure 2).

\section{Authors' Note}

Both parents granted consent for publication of this case report.

\section{Declaration of Conflicting Interests}

The author(s) declared no potential conflicts of interest with respect to the research, authorship, and/or publication of this article.

\section{Funding}

The author(s) received no financial support for the research, authorship, and/or publication of this article.

\section{Supplemental Material}

Supplemental material for this article is available online. 


\section{References}

1. Wood P. Diseases of the Heart and Circulation. 2nd ed. Philadelphia, PA: Lippincott; 1956:457-458.

2. Aminololama-Shakeri S, Wootton-Gorges SL, Pretzlaff RK, Reyes M, Moore EH. Right-sided superior vena cava draining into the left atrium: a rare anomaly of systemic venous return. Pediatr Radiol. 2007;37(3): 317-320.

3. Van Praagh S, Geva T, Lock JE, Nido PJ, Vance MS, Van Praagh R. Biatrial or left atrial drainage of the right superior vena cava: anatomic, morphogenetic, and surgical considerations - report of three new cases and literature review. Pediatr Cardiol. 2003;24(4): 350-363.
4. Braudo M, Beanlands DS, Trusler G. Anomalous drainage of the right superior vena cava into the left atrium. Can Med Assoc J. 1968;99(14): 715-719.

5. Warden HE, Gustafson RA, Tarnay TJ, Neal WA. An alternative method for repair of partial anomalous pulmonary venous connection to the superior vena cava. Ann Thorac Surg. 1984;38(6): 601-605.

6. Recto MR, Sobczyk WL, Yeh T. Right superior vena cava draining predominantly into the left atrium causing cyanosis in a young child. Pediatr Cardiol. 2004;25(2): 163-164.

7. Yerebakan C, Frank L, Hibino N, Jonas RA. Partial anomalous pulmonary venous connection with anomalous connection of the superior vena cava to the left atrium. J Thorac Cardiovasc Surg. 2012;144(1): e1-e3. 